\title{
A new expression for sums of powers of integers in terms of Stirling Numbers of the second kind
}

\author{
Sumit Kumar Jha \\ IIIT-Hyderabad, India \\ Email: kumarjha.sumit@research.iiit.ac.in
}

June 1, 2019

\begin{abstract}
In this brief note, we derive an expression for sums of powers of integers in terms of Stirling Numbers of the second kind. To the best of our knowledge, this expressions is new.

Keywords: Sum of powers of integers; Stirling Numbers of the second kind; Eulerian Numbers; Riemann Zeta Function; Hurwitz Zeta Function AMS Classification: Primary: 11B73; Secondary: 11B37
\end{abstract}

Let $S_{r}(n)=\sum_{k=1}^{n} k^{r}$. Then, the following formula is well-known [1]

$$
S_{r}(n)=\sum_{k=1}^{r} k !\left\{\begin{array}{l}
r \\
k
\end{array}\right\}\left(\begin{array}{l}
n+1 \\
k+1
\end{array}\right) \text {, }
$$

where $\left\{\begin{array}{l}r \\ k\end{array}\right\}$ denote Stirling number of the second kind.

We prove the following here.

Theorem 1. We have

$$
S_{r}(n)=(-1)^{r} \sum_{k=1}^{r}(-1)^{k} \cdot k !\left\{\begin{array}{l}
r \\
k
\end{array}\right\}\left(\begin{array}{l}
n+k \\
k+1
\end{array}\right),
$$

where $\left\{\begin{array}{l}r \\ k\end{array}\right\}$ denote the Stirling numbers of the second kind.

Proof. To prove (2), we use the following result

$$
\frac{\sin n \pi}{\pi} \int_{0}^{\infty} x^{n-1} \frac{\mathbf{L i}_{s}(-x)}{1+x} d x=\zeta(s)-\zeta(s, 1-n),
$$

where $\mathbf{L i}_{s}(-x)$ denotes the Polylogarithm function, $\zeta(s)$ is the Riemann zeta function, and $\zeta(s, 1-n)$ is the Hurwitz zeta function. The above integral is valid for all $s \in \mathbb{C} \backslash\{1\}$, and $n \in \mathbb{R}$. This integral can be obtained from formula 3.2.1.6 in the book [2]. 
To prove (2), we use the following representation from the note [3]

$$
\mathbf{L i}_{-r}(-x)=\sum_{k=1}^{r} k !\left\{\begin{array}{l}
r \\
k
\end{array}\right\}\left(\frac{1}{1+x}\right)^{k+1}(-x)^{k},
$$

which can be easily proved using induction on $r$.

To deduce (2), we follow the steps below

$$
\begin{array}{r}
\zeta(-r)-\zeta(-r, 1-n)=\frac{\sin n \pi}{\pi} \int_{0}^{\infty} x^{n-1} \frac{\mathbf{L i}_{-r}(-x)}{1+x} d x \\
=\frac{\sin n \pi}{\pi} \sum_{k=1}^{r}(-1)^{k} \cdot k !\left\{\begin{array}{l}
r \\
k
\end{array}\right\} \int_{0}^{\infty} \frac{x^{n-1+k}}{(1+x)^{k+2}} d x \\
=\frac{\sin n \pi}{\pi} \sum_{k=1}^{r}(-1)^{k} \cdot k !\left\{\begin{array}{l}
r \\
k
\end{array}\right\} \frac{\Gamma(n+k) \Gamma(2-n)}{\Gamma(k+2)} \\
=\frac{\sin n \pi \cdot \Gamma(2-n)}{\pi} \sum_{k=1}^{r}(-1)^{k} \cdot k !\left\{\begin{array}{l}
r \\
k
\end{array}\right\} \frac{\Gamma(n+k)}{\Gamma(k+2)} \\
=-\frac{1}{\Gamma(n-1)} \sum_{k=1}^{r}(-1)^{k} \cdot k !\left\{\begin{array}{l}
r \\
k
\end{array}\right\} \frac{\Gamma(n+k)}{\Gamma(k+2)} \\
=-\sum_{k=1}^{r}(-1)^{k} \cdot k !\left\{\begin{array}{l}
r \\
k
\end{array}\right\}\left(\begin{array}{c}
n+k-1 \\
k+1
\end{array}\right),
\end{array}
$$

where $\Gamma(\cdot)$ is the Gamma function.

Now, also,

$$
\begin{array}{r}
\zeta(-r)-\zeta(-r, 1-n)=\frac{(-1)^{r} B_{r+1}}{r+1}-\frac{B_{r+1}(1-n)}{r+1} \\
=\frac{(-1)^{r} B_{r+1}}{r+1}-\frac{(-1)^{r+1} B_{r+1}(n)}{r+1} \\
=(-1)^{r+1} S_{r}(n-1),
\end{array}
$$

here $B_{r+1}$, and $B_{r+1}(n)$ denote Bernoulli numbers, and Bernoulli polynomials respectively. Thus, we complete the proof of (2).

\section{References}

1. R. L. Graham and D. E. Knuth and O. Patashnik, Concrete Mathematics: A Foundation for Computer Science, Addison-Wesley, 1994.

2. Handbook of Mellin Transforms by Yu. Brychkov, O. Marichev, N, Savischenko, 2019.

3. Stirling Numbers and Polylogarithms by Steven E. Landsburg. Link: http://www.landsburg.com/query.pdf 